\title{
Heterogeneidade de (Co)variância para as Produções de Leite e de Gordura entre Vacas Puras e Mestiças da Raça Gir
}

\section{Claudio Napolis Costa ${ }^{1,2}$, Mário Luiz Martinez ${ }^{1,2}$, Rui da Silva Verneque ${ }^{1,2}$, Roberto Luiz Teodoro ${ }^{1,2}$, Ivan Luz Ledic ${ }^{1}$}

\begin{abstract}
RESUMO - Registros de produção de leite e de gordura de 5086 vacas puras e mestiças da raça Gir de rebanhos de diferentes Estados do Brasil, ajustados para idade adulta, rebanho, ano e estação de parto, foram utilizados para estimar componentes de (co)variância por análises bicaráter e multicaráter com modelo animal pelo método REML. O modelo multicárater para a predição de valores genéticos (VG) para as produções de leite (PL) e de gordura (PG) de vacas puras e mestiças foi comparado com o modelo bicárater (leite e gordura) como alternativa para o ajuste da heterogeneidade de variância da PL (características distintas) entre grupos genéticos (vacas puras e mestiças). As estimativas dos componentes de (co)variância para as produções de leite e de gordura foram heterogêneas entre vacas puras e mestiças. As variâncias genéticas aditivas para leite $\left(115.536,4 \mathrm{~kg}^{2}\right)$ e para gordura $\left(214,8 \mathrm{~kg}^{2}\right)$ de vacas puras foram o triplo das estimadas para leite $\left(39.080,4 \mathrm{~kg}^{2}\right)$ e para gordura $\left(60,8 \mathrm{~kg}^{2}\right)$ em vacas mestiças. As correlações genéticas entre as produções de leite e de gordura variaram de 0,73 a 0,99 e foram maiores para as produções de leite $(0,86)$ do que para as produções de gordura $(0,76)$ entre vacas puras e mestiças. As herdabilidades $\left(\mathrm{h}^{2}\right)$ das produções de leite e de gordura foram respectivamente maiores nas vacas puras $(0,23$ e 0,20$)$ do que nas vacas mestiças $(0,08$ e 0,07$)$. As estimativas de correlação genética $\left(r_{g}\right)$ entre as produções de leite e de gordura foram 0,95 e 0,99 , respectivamente para as vacas puras e mestiças. A estimativa de covariância genética entre as produções de leite e de gordura de vacas puras $\left(4.742,9 \mathrm{~kg}^{2}\right)$ foi o triplo da obtida para vacas mestiças. As estimativas dos componentes de variância genética para as produções de leite (99.104,92 $\left.\mathrm{kg}^{2}, \mathrm{~h}^{2}=0,20\right)$ e de gordura $\left(181,21 \mathrm{~kg}^{2}, \mathrm{~h}^{2}=0,18\right)$ do modelo bicaráter corresponderam, respectivamente, a 85,9 e $84,4 \%$ dos valores obtidos em vacas puras da raça Gir com o modelo multicaráter. A estimativa de covariância genética foi $4.071,14 \mathrm{~kg}^{2}$, com r $\mathrm{r}_{\mathrm{g}}$ gual a 0,96 entre as produções de leite e de gordura. As correlações de ordem entre os VG para PL de animais Gir e VG para PL e PG de animais mestiços estimados com o modelo multicaráter e os VG para PL e PG obtidos com o modelo bicaráter foram superiores a 0,96. Alterações observadas na ordem de classificação de touros e vacas indicam maior precisão na seleção e maior potencial de progresso genético com a utilização do modelo multicaráter nas avaliações genéticas.
\end{abstract}

Palavras-chave: heterogeneidade de variância, parâmetros genéticos, avaliação genética, seleção

\section{Heterogeneity of (Co)variance for Milk and Fat Yields Between Purebred and Crossbred Gir Cows}

\begin{abstract}
First lactation milk and fat yields of 5086 purebred and crossbred Gir cows were used to estimate genetic components of (co)variance using multivariate and bivariate animal models solved by a REML derivative-free algorithm. Both models were used to predict breeding values (EBV) for milk (MY) and fat (FY) yields of purebred and crossbred Gir cows. In the multivariate model it was assumed yields from each genetic group as different traits to account for heterogeneous phenotypic variance for milk yield. This model was compared to a bivariate model assuming homogeneous genetic expression between genetic groups (purebred and crossbred cows). Additive genetic and residual variances were heterogeneous for genetic groups. Genetic variances for milk $\left(115,536.4 \mathrm{~kg}^{2}\right)$ and fat $\left(214.8 \mathrm{~kg}^{2}\right)$ of purebred Gir cows were approximately three folds larger than estimates for milk $\left(39,080.4 \mathrm{~kg}^{2}\right)$ and fat $\left(60.8 \mathrm{~kg}^{2}\right)$ of crossbred cows. Genetic correlations ranged from 0.73 to 0.99 between milk and fat yields and were larger for milk yields ( 0.86 ) than for fat yields (0.76) between different genetic groups. Heritability $\left(\mathrm{h}^{2}\right)$ for milk and fat yields were larger in purebred $(0.23$ and 0.20 respectively) than in crossbred cows ( 0.08 and 0.07 respectively). Genetic correlation between milk and fat yields were 0.95 and 0.99 for purebred and crossbred Gir cows respectively. Genetic variances for milk $\left(99,104.92 \mathrm{~kg}^{2}, \mathrm{~h}^{2}=0.20\right)$ and fat $\left(181.21 \mathrm{~kg}^{2}, \mathrm{~h}^{2}=0.18\right)$ estimated from the bivariate model were respectively $85.9 \%$ and $84.4 \%$ of the corresponding estimates for purebred Gir cows from the mutivariate model. Genetic covariance and correlation between milk and fat yields estimated from the bivariate model were respectively 4,071.14 $\mathrm{kg}^{2}$ and 0.96. Rank correlation estimates were larger than 0.96 between EBV for MY of Gir cows and EBV for MY and FY of crossbred cows and EBV for MY and FY from the bivariate model. Changes in ranking of sires and cows suggest higher selection accuracy and greater potential of genetic progress by application of a multiple trait animal model for genetic evaluations.
\end{abstract}

Key Words: heterogeneity of variance, genetic parameter, selection, genetic evaluation

\footnotetext{
${ }_{1}^{1}$ Pesquisador da Embrapa Gado de Leite. Rua Eugênio do Nascimento 610, 36038-330, Juiz de Fora-MG. (cnc8@cnpgl.embrapa.br) ${ }^{2}$ Bolsista CNPq.
} 


\section{Introdução}

A avaliação genética constitui-se em um procedimento essencial para obtenção de informações que orientam os produtores na identificação e no acasalamento de animais de melhor mérito genético para a geração de progresso genético nos programas de seleção. As avaliações genéticas para as características sob seleção necessitam dos respectivos componentes de (co)variância, cujos valores determinam as estimativas de herdabilidade e das correlações genéticas entre as características, a precisão das predições dos valores genéticos e o progresso genético esperado na população sob seleção. Além da precisão das estimativas dos componentes de (co)variância, a adequabilidade das pressuposições dos modelos à natureza das informações disponíveis também é um fator determinante da precisão de valores genéticos preditos (VG) para animais de uma população sob seleção.

Nos programas de seleção de bovinos de leite, a existência de heterogeneidade de variância, devido aos efeitos de escala associados a níveis de produção, variabilidade dos rebanhos e mesmo composição racial dos animais, tem sido amplamente relatada e suas implicações na precisão das predições dos valores genéticos dos animais, investigadas (Vinson, 1987; Boldman \& Freeman, 1990; Dong \& Mao, 1990; Costa, 1999). Nesse contexto, vários procedimentos têm sido avaliados para o ajuste da heterogeneidade de variância (Wiggans \& VanRaden, 1991; Meuwissen et al., 1996; Robert-Granié et al., 1999).

No Brasil, os programas de seleção para a produção de leite nas raças zebuínas têm a participação de rebanhos puros e mestiços (Verneque et al., 2000; Machado \& Costa, 2000), o que caracteriza dois tipos de progênies para os touros em teste: puras e mestiças. A participação de animais mestiços contribui para formação de grupos de progênies de maior tamanho efetivo, o que permite maior precisão nas predições de valor genético dos animais. Todavia, a presença de heterogeneidade de variância entre animais de diferentes grupos raciais pode comprometer a precisão das predições de VG, reduzindo o progresso genético dos programas de seleção. Neste particular, Martinez et al. (2000) avaliaram a relação entre os valores genéticos de touros Gir preditos por modelos unicaráter para as produções de leite de suas progênies puras e mestiças. Os resultados indicaram que o melhor critério de seleção seria um índice combinan- do os valores genéticos obtidos em análises distintas para os dois tipos de progênie. Alternativamente, poder-se-ia ajustar um modelo multicaráter, considerando-se os desempenhos em cada grupo genético como características distintas. Este procedimento permite ajustar diferentes variâncias para cada tipo de progênie (Gianola, 1986) e, de forma mais objetiva, predizer o valor genético de animais de diferentes grupos genéticos utilizando-se das correlações genéticas entre as "distintas" características. O critério de seleção para touros jovens da raça Gir, em teste de progênie, por meio da analise simultânea dos registros de desempenho de suas progênies puras e mestiças, seria o VG predito do desempenho das progênies puras, uma vez que o objetivo principal é a realização de progresso genético na raça.

Este estudo foi conduzido com o objetivo de avaliar a presença da heterogeneidade de variância para as produções de leite e de gordura em animais puros e mestiços da raça Gir e avaliar o modelo multicaracter (leite e gordura como características distintas entre dois grupos genéticos), comparativamente ao modelo bicaracter (leite e gordura como características comuns ou iguais para os grupos genéticos), como alternativa para ajuste da heterogeneidade de variância da produção de leite e de gordura e sua aplicação na seleção de touros e vacas da raça Gir.

\section{Material e Métodos}

Foram utilizados 5086 registros de produção de leite e de gordura da primeira lactação de vacas puras da raça Gir e mestiças filhas de touros Gir, de rebanhos participantes do Programa Nacional de Melhoramento da raça realizado por meio do Teste de Progênie de touros jovens coordenado pela Embrapa Gado de Leite em convênio com a Associação Brasileira dos Criadores de Gir Leiteiro - ABCGIL. Foram incluídos os registros de produção de leite com porcentagem de gordura entre 3 e $9 \%$ e descartadas as classes de rebanho-ano com menos de três observações e touros com menos de duas progênies. $\mathrm{O}$ número total de touros incluídos neste estudo foi de 399, dos quais 58 apresentaram progênie em rebanhos puros e mestiços da raça Gir. Os animais mestiços constituíram quatro classes de composição racial: 1/2, 3/4, 5/8 e 7/8 de contribuição da raça Gir.

As produções de leite e de gordura até 305 dias ajustadas para idade adulta, obtidas em animais de

R. Bras. Zootec., v.33, n.3, p.555-563, 2004 
diferentes composições raciais (Gir e mestiços Gir, doravante denominados grupos genéticos - GG) foram consideradas características diferentes, e analisadas por um modelo multicaráter, que incluiu os efeitos fixos de rebanho-ano, época de parto (outubro a março e abril a setembro), idade da vaca como covariável com componentes linear e quadrático e composição racial (somente para os animais mestiços) e os efeitos aleatórios de animal e resíduo.

Em notação matricial, o modelo é representado por:

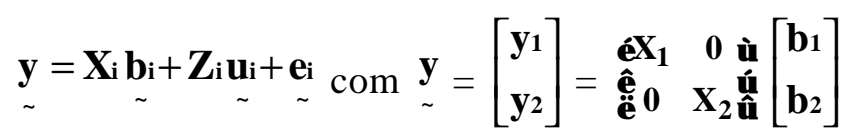
$+\left[\begin{array}{cc}\mathbf{Z}_{1} & \mathbf{0} \\ \mathbf{0} & \mathbf{Z}_{2}\end{array}\right]\left[\begin{array}{l}\mathbf{u}_{1} \\ \mathbf{u}_{2}\end{array}\right]+\left[\begin{array}{l}\mathbf{e}_{1} \\ \mathbf{e}_{2}\end{array}\right]$,

em que $\mathbf{y}$ é o vetor de observações das produções de leite e de gordura nos GG (1=puros; $\mathbf{2}=$ =mestiços); $\mathbf{X i}$ e $\mathbf{Z i}$ são as matrizes de incidência para os efeitos fixos e aleatórios, respectivamente, em cada GG; $\underset{\sim}{\mathbf{b}_{\mathbf{i}}}, \underset{\sim}{\mathbf{u}_{\mathbf{i}}}$ e $\mathbf{e}_{\sim}^{\mathbf{i}}$ são, respectivamente, os vetores de efeitos fixos e aleatórios de animal e de resíduo associados a cada característica de produção em cada GG.

Foi assumido que:

$$
\begin{gathered}
{\left[\begin{array}{l}
\mathbf{u} \\
\mathbf{e}
\end{array}\right] \sim N\left(\left[\begin{array}{l}
\mathbf{0} \\
\mathbf{0}
\end{array}\right] \quad\left[\begin{array}{ll}
\mathbf{G} & \mathbf{0} \\
\mathbf{0} & \mathbf{R}
\end{array}\right]\right) \text { para } \mathbf{G}=\mathbf{A} \otimes \mathbf{G}_{\mathbf{0}} \text { sendo }} \\
\mathbf{G o}=\left[\begin{array}{cccc}
\boldsymbol{\sigma}_{11}^{2} & \boldsymbol{\sigma}_{11 \mathrm{~g} 1} & \boldsymbol{\sigma}_{1112} & \boldsymbol{\sigma}_{\mathbf{1 1 g} 2} \\
& \boldsymbol{\sigma}_{\mathrm{g} 1}^{2} & \boldsymbol{\sigma}_{\mathrm{g} 122} & \boldsymbol{\sigma}_{\mathrm{g} 1 \mathrm{~g} 2} \\
& & \boldsymbol{\sigma}_{12}^{2} & \boldsymbol{\sigma}_{\mathbf{1 2} 2} \\
& & \boldsymbol{\sigma}_{\mathrm{g} 2}^{2}
\end{array}\right]=\operatorname{var}(\mathbf{u}),
\end{gathered}
$$

em que $\sigma_{\mathrm{li}}^{2}$ e $\sigma_{\mathrm{gi}}^{2}$ são os componentes de variância genética aditiva para as produções de leite e de gordura, respectivamente; $\sigma_{\text {ligi }}$ é a covariância genética entre as produções de leite e de gordura em cada GG; $\sigma_{\text {lilj }}$ e $\sigma_{\text {gigj }}$ são as covariâncias genéticas entre GG para as produções de leite e de gordura, respectivamente; e $\sigma_{\text {lilj }} \mathrm{e} \sigma_{\text {gigj }}$ são as covariâncias genéticas entre GG para as produções de leite e de gordura; e A é a matriz do numerador dos coeficientes de parentesco entre os animais.

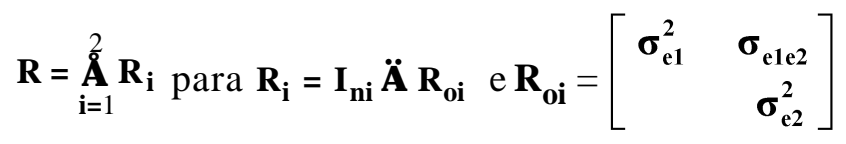$$
=\operatorname{var}(\mathbf{e}) \text { em que } \oplus \mathrm{e} \otimes \text { são, respectivamente, }
$$

operadores de soma e produto direto; $\sigma_{\mathbf{e} 1}^{2}$ e $\sigma_{\mathbf{e} 2}^{2}$ são os componentes de variância residual para as produções de leite e de gordura, respectivamente; $\sigma_{\text {e1e2 }}$ é a covariância residual entre as produções de leite e de gordura em cada GG; e $\mathbf{I}_{\mathbf{n i}}$ é uma matriz identidade de ordem igual ao numero de registros em cada GG.

Para o modelo bicaráter assumiu-se homogeneidade de expressão genética entre grupos genéticos, que em sua forma mais simples se expressa por $\mathbf{y}=\mathbf{X} \mathbf{\sim}+\mathbf{Z}+\underset{\sim}{\mathbf{u}}+\underset{\sim}{\mathbf{e}}$ em que $\mathbf{y}$ é o vetor de observações das produções de leite é de gordura, b, u, e são, respectivamente, os vetores de efeito fixõs (os mesmos efeitos considerados no modelo multicaráter e composição racial com subclasses: puros, 1/2, 3/4, $5 / 8$ e 7/8 da raça Gir); efeito aleatório de animal e resíduo; $\mathbf{X}$ e $\mathbf{Z}$ são matrizes de incidência dos efeitos fixos e aleatórios, respectivamente.

Foi assumido que:

$$
\left[\begin{array}{l}
\mathbf{u} \\
\mathbf{e}
\end{array}\right] \sim N\left(\left[\begin{array}{l}
\mathbf{0} \\
\mathbf{0}
\end{array}\right] \quad\left[\begin{array}{ll}
\mathbf{G} & \mathbf{0} \\
\mathbf{0} & \mathbf{R}
\end{array}\right]\right) \text {, sendo }
$$

$\mathbf{G}=\mathbf{A} \otimes \mathbf{G o}, \quad \mathbf{G o}=\left[\begin{array}{cc}\boldsymbol{\sigma}_{1}^{2} & \boldsymbol{\sigma}_{\mathrm{lg}} \\ & \boldsymbol{\sigma}_{\mathrm{g}}^{2}\end{array}\right]=\operatorname{var}(\mathbf{u}), \quad \mathrm{e}$ $\mathbf{R}=\mathbf{I}_{\mathrm{ni}} \otimes \mathbf{R o}, \mathbf{R o}=\left[\begin{array}{cc}\boldsymbol{\sigma}_{\mathrm{el}}^{2} & \boldsymbol{\sigma}_{\mathrm{elg}} \\ & \boldsymbol{\sigma}_{\mathrm{eg}}^{2}\end{array}\right]=\operatorname{var}(\mathbf{e})$, em que $\sigma_{\text {el }}^{2}$ e $\sigma_{\text {eg }}^{2}$ são, respectivamente, as variâncias residuais para as produções de leite e de gordura; $\sigma_{\text {elg }}$, a covariância residual entre as produções medidas em um mesmo animal; e $\mathbf{I}_{\text {ni }}$, uma matriz identidade de ordem igual ao número de registros utilizados na análise.

Os componentes de (co)variância foram estimados usando-se os programas MTDFREML desenvolvidos por Boldman et al. (1995). A convergência do processo iterativo foi definida quando a variância dos valores de $-2 \times$ logaritmo do valor da função de máxima verossimilhança (- $2 \log \ell$ ) era inferior a $10^{-8}$. As análises foram repetidas com valores de convergência de análises anteriores até que o valor da terceira casa decimal de $\mathbf{- 2} \log \ell$ não se modificasse. A estimativa de correlação genética entre as produções de leite e de gordura e suas respectivas herdabilidades foram obtidas por: $\hat{\mathbf{r}}_{\mathrm{g}}=\hat{\boldsymbol{\sigma}}_{\mathrm{g}} /\left(\hat{\boldsymbol{\sigma}}_{\mathbf{l}}^{2} \times \hat{\boldsymbol{\sigma}}_{\mathrm{g}}^{2}\right)^{0,5}$, $\hat{\mathbf{h}}_{\mathrm{l}}^{2}=\hat{\boldsymbol{\sigma}}_{\mathrm{l}}^{2} /\left(\hat{\boldsymbol{\sigma}}_{\mathrm{l}}^{2}+\hat{\boldsymbol{\sigma}}_{\mathrm{el}}^{2}\right)$ e $\hat{\mathbf{h}}_{\mathrm{g}}^{2}=\hat{\boldsymbol{\sigma}}_{\mathrm{g}}^{2} /\left(\hat{\boldsymbol{\sigma}}_{\mathrm{g}}^{2}+\hat{\boldsymbol{\sigma}}_{\mathrm{eg}}^{2}\right)$.

As médias para os VG preditos para as produ-

R. Bras. Zootec., v.33, n.3, p.555-563, 2004 
ções de leite e de gordura e respectivas confiabilidades para os modelos multicaráter e bicaráter foram obtidas usando-se o SAS ${ }^{\circledR}$. As comparações entre as classificações de touros e vacas foram realizadas mediante estimativas de correlação de ordem usando-se o procedimento Proc rank do mesmo sistema.

\section{Resultados e Discussão}

As médias, desvios-padrão (DP) e respectivos número de lactações para as produções de leite e de gordura de animais da raça Gir e seus mestiços são apresentados na Tabela 1. Observa-se que os animais mestiços contribuem com, aproximadamente, 24 e $27 \%$ das registros das produções de leite e de gordura, respectivamente. Também é evidente a maior produção de leite e variabilidade (DP) dos animais 5/8 e $1 / 2$ em relação aos outros mestiços, com maior contribuição da raça Gir na composição racial. No conjunto, as médias de produção dos mestiços são inferiores àquelas correspondentes aos animais Gir, mas os respectivos DPs praticamente não diferem.

As estimativas dos componentes de variância genética para a produção de leite $\left(99.104,92 \mathrm{~kg}^{2}\right.$, herdabilidade $=0,20)$ e de gordura $\left(181,21 \mathrm{~kg}^{2}\right.$, herdabilidade $=0,18$ ) do modelo bicaráter corresponderam, respectivamente, a 85,9 e $84,4 \%$ dos valores obtidos em animais puros da raça Gir com o modelo multicaráter. A estimativa de covariância genética para este modelo foi $4.071,14 \mathrm{~kg}^{2}$, com correlação igual a 0,96 entre as produções de leite e de gordura.

As estimativas dos componentes de variância e covariância genética para as produções de leite e de gordura nos animais mestiços corresponderam, respectivamente, a 33,8;28,3 e 32,3\% das estimativas obtidas nos animais puros (Tabela 2). Para as (co)variâncias residuais estas proporções foram 81,8; 113,2 e $94,7 \%$, respectivamente. Tais diferenças indicam a heterogeneidade de variâncias entre os dois grupos genéticos e resultaram em valores de herdabilidade maiores para as produções de leite e de gordura nos animais puros do que nos animais mestiços $(0,23$ e 0,20 e 0,08 e 0,07 , respectivamente) e de correlação genética entre as produções de leite e de gordura maior nos animais mestiços $(0,99)$ do que nos animais puros $(0,95)$.

As estimativas das correlações genéticas para as produções de leite e de gordura entre os GG foram 0,73 e 0,87 . A estimativa de correlação genética para as produções de leite entre GG foi maior $(0,86)$ do que a obtida para as produções de gordura $(0,76)$. Tais estimativas sugerem que se espera maior mudança da classificação no valor genético de touros entre os GG para a produção de gordura do que para a produção de leite.

A heterogeneidade de variâncias entre os GG significa que as diferenças entre touros nas progênies mestiças são menores do que aquelas observadas nas progênies puras (Tabela 3). Este efeito de escala é

Tabela 1 - Média e desvio-padrão (DP) e respectivo número de lactações para as produções de leite e de gordura de animais da raça Gir e mestiços

Table 1 - Average, standard deviation (SD) for milk and fat yields and respective number of lactation records by genetic group of purebred and crossbred Gir cows

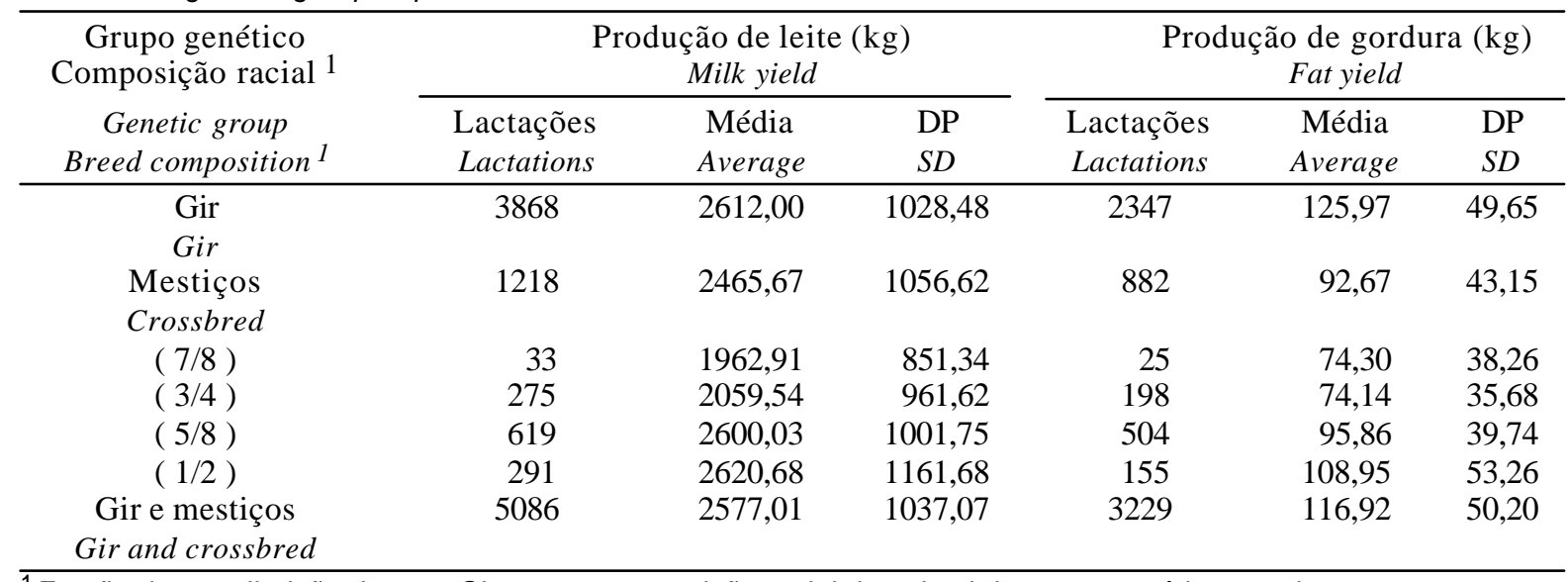

${ }^{1}$ Fração de contribuição da raça Gir para a composição racial do animal do grupo genético mestiços.

${ }^{1}$ Ratio of Gir contribution to the animal breed composition in the crossbred genetic group. 
importante se as progênies de touros não são igualmente distribuídas entre os rebanhos puros e mestiços (Vinson, 1987; Boldman \& Freeman, 1990; Costa, 1999), pois touros com maior número de progênies mestiças tendem a ter os seus valores genéticos subestimados, se a heterogeneidade de variância não for considerada nas avaliações genéticas.
Na Tabela 4, são apresentadas as médias, os desvios-padrão e respectivas confiabilidades dos VGs para as produções de leite e de gordura para touros e vacas, por modelo de análise. Observa-se que os VGs para a produção de leite foram maiores para animais puros $(42,86$ e 70,57) que aqueles observados para animais mestiços $(22,19$ e 37,24$)$ no modelo

Tabela 2 - Estimativas de (co)variância genética/residual $\left(\mathrm{kg}^{2}\right)$ para as produções de leite e de gordura com respectivos valores de herdabilidade e correlações genéticas (entre parêntesis) para vacas puras Gir (sub-matriz triangular esquerda superior) e mestiças Gir (sub-matriz triangular direita inferior) obtidas com análise multicarater com um modelo animal

Table 2 - Estimates of additive genetic and residual (co)variance components and respective estimates of genetic correlation and heritability (in parenthesis) for milk and fat yields of purebred and crossbred Gir cows

\begin{tabular}{|c|c|c|c|c|}
\hline \multirow{2}{*}{$\begin{array}{l}\text { Grupo genético } 1 \\
\text { Genetic group }^{1} \\
\text { Produção } \\
\text { Yield } \\
\end{array}$} & \multicolumn{2}{|l|}{$\begin{array}{l}\text { Gir } \\
\text { Gir }\end{array}$} & \multicolumn{2}{|c|}{$\begin{array}{l}\text { Mestiças } \\
\text { Crossbred }\end{array}$} \\
\hline & $\begin{array}{l}\text { Leite } \\
\text { Milk }\end{array}$ & $\begin{array}{c}\text { Gordura } \\
\text { Fat }\end{array}$ & $\begin{array}{l}\text { Leite } \\
\text { Milk }\end{array}$ & $\begin{array}{c}\text { Gordura } \\
\text { Fat }\end{array}$ \\
\hline Gir & & & & \\
\hline $\begin{array}{l}\text { Leite } \\
\text { Milk }\end{array}$ & $\begin{array}{c}115.536,4 / 380.603,3 \\
(0,23)\end{array}$ & $\begin{array}{c}4.742,9 / 16.681,0 \\
(0,95)\end{array}$ & $\begin{array}{c}58.095,4 / 0 \\
(0,86)\end{array}$ & $\begin{array}{l}2.308,1 / 0 \\
(0,87)\end{array}$ \\
\hline $\begin{array}{l}\text { Gordura } \\
\text { Fat }\end{array}$ & & $\begin{array}{c}214,8 / 871,2 \\
(0,20)\end{array}$ & $\begin{array}{l}2.117,2 / 0 \\
\quad(0,73)\end{array}$ & $\begin{array}{l}87,4 / 0 \\
(0,76)\end{array}$ \\
\hline $\begin{array}{l}\text { Mestiços } \\
\text { Crossbred }\end{array}$ & & & & \\
\hline $\begin{array}{l}\text { Leite } \\
\text { Milk }\end{array}$ & & & $\begin{array}{c}39.080,4 / 465.320,7 \\
(0,08)\end{array}$ & $\begin{array}{c}1.534,1 / 17.605,8 \\
(0,99)\end{array}$ \\
\hline $\begin{array}{l}\text { Gordura } \\
\text { Fat }\end{array}$ & & & & $\begin{array}{c}60,8 \text { / 769,3 } \\
(0,07)\end{array}$ \\
\hline
\end{tabular}

${ }^{1}$ Gir = animais puros, Mestiços = conjunto de todas as composições raciais.

${ }^{1}$ Gir = purebred animals; Crossbred $=$ all crossbred groups toghether .

Tabela 3 - Média, desvio-padrão (DP) e correlação de ordem ( $r$ ) entre os valores genéticos preditos para a produção de leite (VGL) e de gordura (VGG) pelos dois modelos ajustados, para animais de raça Gir e seus mestiços

Table 3 - Average, standard deviation (SD) and rank correlation ( $r$ ) between predicted breeding value for milk (VGL) and fat (VGG) yields for purebred and crossbred Gir cows in the two models of analysis

\begin{tabular}{|c|c|c|c|c|c|c|}
\hline \multicolumn{2}{|c|}{$\begin{array}{l}\text { Modelo de análise } \\
\text { Model of analysis }\end{array}$} & \multicolumn{2}{|c|}{ VGL } & \multicolumn{2}{|c|}{ VGG } & \multirow[b]{2}{*}{$r$} \\
\hline & & \multirow{2}{*}{$\begin{array}{c}\text { Média } \\
\text { Average }\end{array}$} & $\begin{array}{l}\mathrm{DP} \\
S D \\
\end{array}$ & $\begin{array}{c}\text { Média } \\
\text { Average }\end{array}$ & $\begin{array}{l}\mathrm{DP} \\
S D\end{array}$ & \\
\hline $\begin{array}{l}\text { Bicaráter } \\
\text { Bivariate }\end{array}$ & & & 143,62 & & 6,00 & 0,982 \\
\hline $\begin{array}{l}\text { Multicaráter } \\
\text { Multivariate }\end{array}$ & $\begin{array}{l}\text { Gir } \\
\text { Gir }\end{array}$ & 48,20 & 155,54 & 1,99 & 6,45 & 0,978 \\
\hline & $\begin{array}{l}\text { Mestiços } \\
\text { Crossbred }\end{array}$ & 25,17 & 83,74 & 1,00 & 3,29 & 0,998 \\
\hline
\end{tabular}

R. Bras. Zootec., v.33, n.3, p.555-563, 2004 
multicaráter, ou para os mesmos valores obtidos com o modelo bicaráter $(39,17$ e 64,39). A mesma tendência, com ligeira superioridade, foi observada para a produção de gordura e para as respectivas estimativas de confiabilidade. Estas diferenças refletem a magnitude das diferenças nos componentes de variância de cada grupo genético (em conjunto, ou puros e mestiços da raça Gir).

$\mathrm{Na}$ Tabela 5, são apresentadas as estimativas de correlação de ordem entre os valores genéticos preditos pelos dois modelos. Os valores das correlações foram todos superiores a 0,96 entre a produção de leite de animais Gir com o modelo multicaráter e as produções de leite e de gordura pelo modelo bicarater e para as produções de leite e de gordura dos animais mestiços. As menores estimativas de correlação de ordem foram observadas entre as produções de leite e de gordura de mestiços e a produção de gordura de Gir $(0,90$ e 0,93 , respectivamente) e a produção de gordura pelo modelo bicarater $(0,91$ e 0,94 , respectivamente).

Rodriguez-Almeida et al. (1995) observaram que a pressuposição de variâncias homogêneas entre grupos genéticos é errada, no contexto das avaliações genéticas de animais de diferentes composições raciais. A análise detalhada entre os dez melhores touros Gir classificados pelo VG para produção de leite pelo modelo multicaráter, comparativamente ao modelo bicaráter, não apresentou alteração entre os três primeiros classificados. Todavia, observaram-se seis mudanças, sendo cinco até três posições na

Tabela 4 - Média e desvio-padrão (DP) para valores genéticos preditos e respectivas confiabilidades $\left(\mathbf{r t}_{\mathbf{i}}\right)^{2}$ para a produção de leite $(V G L)^{1}$ e de gordura (VGG) para touros e vacas, pelos dois modelos de análise

Table 4 - Average and standard deviation of predicted breeding values of bulls and cows for milk $(V G L)^{1}$ and fat (VGG) yields and respective accuracy $\left(\boldsymbol{r t}_{i}\right)^{2}$ in the two models of analysis

\begin{tabular}{|c|c|c|c|c|}
\hline \multirow[t]{2}{*}{$\begin{array}{c}\text { Modelo } \\
\text { Model }\end{array}$} & \multicolumn{2}{|c|}{$\begin{array}{c}\text { Touros } \\
\text { Bulls }\end{array}$} & \multicolumn{2}{|c|}{$\begin{array}{l}\text { Vacas } \\
\text { Cows }\end{array}$} \\
\hline & Média & DP & Média & $\begin{array}{l}\text { DP } \\
S D\end{array}$ \\
\hline \multicolumn{5}{|l|}{ Bicaráter } \\
\hline \multicolumn{5}{|l|}{ Bivariate } \\
\hline VGL & 39,17 & 173,96 & 64,39 & 168,36 \\
\hline $\mathrm{rt}_{\mathrm{iL}}$ & 0,515 & 0,197 & 0,540 & 0,064 \\
\hline VGG & 1,65 & 7,25 & 2,66 & 7,05 \\
\hline $\mathrm{rt}_{\mathrm{iG}}$ & 0,502 & 0,195 & 0,526 & 0,065 \\
\hline \multicolumn{5}{|l|}{ Multicaráter } \\
\hline Multivariate & & & & \\
\hline $\mathrm{VGL}_{\mathrm{G}}$ & 42,86 & 187,98 & 70,57 & 190,95 \\
\hline $\mathrm{rt}_{\mathrm{iLG}}$ & 0,518 & 0,193 & 0,565 & 0,060 \\
\hline VGL $_{M}$ & 22,19 & 102,04 & 37,24 & 101,34 \\
\hline $\mathrm{rt}_{\mathrm{iLM}}$ & 0,476 & 0,180 & 0,512 & 0,061 \\
\hline $\mathrm{vGG}_{\mathrm{G}}$ & 1,78 & 7,79 & 2,86 & 7,94 \\
\hline $\mathrm{rt}_{\mathrm{iGG}}$ & 0,496 & 0,190 & 0,542 & 0,061 \\
\hline $\mathrm{VGG}_{\mathrm{M}}$ & 0,88 & 3,99 & 1,48 & 3,96 \\
\hline $\mathrm{rt}_{\mathrm{iGM}}$ & 0,472 & 0,177 & 0,507 & 0,058 \\
\hline
\end{tabular}

${ }_{1}^{1} \mathrm{VGL}, \mathrm{VGG}=$ valores genéticos para leite e gordura, respectivamente, preditos por modelo bicarater; $V \mathrm{VL}_{\mathrm{G}}, \mathrm{VGG}_{\mathrm{G}}, \mathrm{VGL}_{\mathrm{M}}, \mathrm{VGG}_{\mathrm{M}}=$ valores genéticos para leite e gordura, respectivamente, para animais puros da raça Gir e seus mestiços, preditos por modelo multicaráter.

$2 \mathrm{rt}_{\mathrm{iL}}, \mathrm{rt}_{\mathrm{iG}}=$ confiabilidade para os valores genéticos para leite e gordura, respectivamente, preditos por modelo bicaráter; $\mathrm{rt}_{\mathrm{iLG}}, \mathrm{rt}_{\mathrm{GGG}}, \mathrm{rt}_{\mathrm{iLM}} \mathrm{rt}_{\mathrm{iGM}}=$ confiabilidade para os valores genéticos para leite e gordura, respectivamente, para animais puros da raça Gir e seus mestiços, preditos por modelo multicaráter.

${ }^{1} G L, V G G=$ breeding values for milk and fat yields respectively, predicted by the bivariate model; $V G L_{G}$ $V G G_{G} V G L_{M}, V G G_{M}=$ breeding values for milk and fat yields, respectively, for purebred and crossbred Gir animals, predicted by the multivariate model.

$2 \mathrm{rt}_{\mathrm{iL}}, \mathrm{rt}_{\mathrm{iG}}=$ accuracy of breeding values for milk and fat yields, respectively, predicted by the bivariate model; $\mathrm{rt}_{\mathrm{iLG}}, \mathrm{rt}_{\mathrm{GG}}, \mathrm{rt}_{\mathrm{iLM}}, \mathrm{rt}_{\mathrm{GGM}}=$ accuracy of breeding values for milk and fat yields respectively, for purebred and crossbred Gir animals, predicted by the multivariate model. 
classificação e uma de cinco posições (de $4^{\mathrm{a}}$ para $9^{\mathrm{a}}$ colocação). Das seis mudanças, quatro foram para melhor e duas para pior classificação. De modo geral, o modelo multicaráter apresentou tendência a melhorias da posição dos touros na ordem de classificação. A melhoria na confiabilidade dos VG esteve associada ao maior número de progênies puras em relação ao número de progênies mestiças e a maior variância genética para os animais puros. Quando as progênies são desigualmente distribuídas entre as classes que determinam a heterogeneidade de variância, as avaliações de reprodutores são enganosas (Garrick et al., 1989). No presente estudo, observou-se queda na classificação e ligeira redução na confiabilidade do VG de touros com maior número de progênies mestiças. Na medida em que as progênies de touros têm distribuição homogênea entre as classes de efeitos causadores da heterogeneidade de variância, o ajuste em si pode ter impacto limitado, conforme observado por Robert-Granié et al. (1999), em estudo semelhante para as avaliações genéticas na França.

No caso das 50 melhores vacas Gir classificadas pelo VG para a produção de leite com o modelo multicaráter, observaram-se 18 alterações positivas

Tabela 5 - Correlações de ordem entre valores genéticos preditos pelos modelos de análise para as produções de leite (VGL) e de gordura (VGG) de vacas puras e mestiças da raça Gir

Table 5 - Rank correlations between breeding values for milk (VGL) and fat (VGG) yields predicted by the two models of analysis

\begin{tabular}{|c|c|c|c|c|c|}
\hline \multirow[t]{2}{*}{$\begin{array}{l}\text { Modelo } \\
\text { Model }\end{array}$} & \multicolumn{5}{|c|}{$\begin{array}{l}\text { Valor genético }{ }^{1} \\
\text { Breeding value }\end{array}$} \\
\hline & VGG & $\mathrm{VGL}_{\mathrm{G}}$ & VGL $_{M}$ & $\mathrm{VGG}_{\mathrm{G}}$ & $\mathrm{VGG}_{\mathrm{M}}$ \\
\hline \multicolumn{6}{|l|}{$\overline{\text { Bicarater }}$} \\
\hline \multicolumn{6}{|l|}{ Bivariate } \\
\hline VGL & 0,98 & 0,98 & 0,97 & 0,95 & 0,98 \\
\hline VGG & & 0,96 & 0,91 & 0,98 & 0,94 \\
\hline \multicolumn{6}{|l|}{ Multicarater } \\
\hline \multicolumn{6}{|l|}{ Multivariate } \\
\hline $\mathrm{VGL}_{\mathrm{G}}$ & & & 0,97 & 0,98 & 0,98 \\
\hline VGL $_{M}$ & & & & 0,90 & 0,99 \\
\hline $\mathrm{vGG}_{\mathrm{G}}$ & & & & & 0,93 \\
\hline
\end{tabular}

${ }^{1} \mathrm{VGL}, \mathrm{VGG}=$ valores genéticos para leite e gordura respectivamente, preditos por modelo bicarater; $V \mathrm{GL}_{\mathrm{G}}, \mathrm{VGG}_{\mathrm{G}}, \mathrm{VGL}_{\mathrm{M}}$, $V G_{M}=$ valores geneticos para leite e gordura respectivamente, para animais puros da raça Gir e seus mestiços, preditos por modelo multicarater.

${ }^{1} V G L, V G G=$ breeding values for milk and fat yields respectively, predicted by the bivariate model; VGL $V_{G} V G G_{G}, V G L M, V G G_{M}=$ breeding values for milk and fat yields respectively, for purebred and crossbred Gir animals, predicted by the multivariate model. (pior classificação) e 24 alterações negativas (melhor classificação). Entre os casos de melhoria, houve oito ocorrências de troca de mais de dez posições na ordem de classificação. Em uma análise mais restrita às dez primeiras vacas, foram observadas alterações de seis posições (cinco com uma posição e uma com duas posições para melhor). Destas seis alterações, três foram para melhor e três para pior posicionamento na ordem geral de classificação. Estas alterações podem implicar em decisões erradas na identificação de vacas elite ou mães de touros (Powell et al., 1983; Boldman \& Freeman, 1990; Ibáñez et al., 1996).

Embora tenham sido observadas alterações na classificação dos animais, verifica-se na Tabela 6 que não houve uma mudança drástica no número de animais que permaneceram na relação dos melhores classificados (dez a cem primeiros colocados, para touros e vacas). Entretanto, na medida em que a relação de animais classificados é maior, observa-se maior participação de novos animais, principalmente entre vacas, o que coincide com a observação de que o ajuste para a heterogeneidade de variância tem maior efeito para vacas do que para touros (Powell et al., 1983). Em síntese, a análise com o modelo multicaráter não altera a relação dos melhores touros (ou seja, não são incluídos outros touros identificados como os melhores), mas a ordem de classificação entre eles, indicando melhoria da precisão na identificação dos melhores entre si.

Na Tabela 7, são apresentadas as correlações de ordem entre os VG para as produções de leite para touros e vacas classificadas pelo nível de confiabilidade das respectivas predições de VG, excetuando-se os animais com confiabilidade igual a zero. Observa-se que a correlação é inferior a 0,99 apenas para touros com nível de confiabilidade maior que 0,70 . À medida

Tabela 6 - Número de touros e de vacas da raça Gir na classificação topcom o modelo bicarater que permanecem na classificação top com o modelo multicarater

Table 6 - Number of bulls and cows of the top ranking of the bivariate model remaining in the top ranking with the multivariate model

\begin{tabular}{ccc}
\hline $\begin{array}{c}\text { Classificação } \\
\text { Ranking }\end{array}$ & $\begin{array}{c}\text { Touros } \\
\text { Bulls }\end{array}$ & $\begin{array}{c}\text { Vacas } \\
\text { Cows }\end{array}$ \\
\hline 10 & 09 & 09 \\
25 & 23 & 23 \\
50 & 47 & 44 \\
100 & 91 & 88 \\
\hline
\end{tabular}

R. Bras. Zootec., v.33, n.3, p.555-563, 2004 
que maior confiabilidade resulta de maior número e melhor distribuição da progênie entre rebanhos, este valor pode ser indicação de que os touros com maior número de progênie não as têm igualmente distribuídas entre os grupos genéticos (ou rebanhos puros e mestiços). Esta redução confirma a importância da distribuição adequada da progênie, pois, quanto maior o número de filhas e menor o equilíbrio em sua distribuição entre os grupos genéticos, maior a chance de se obter uma classificação errada de touros. Portanto, o ajuste é necessário para a correta classificação de touros, particularmente aqueles de VG mais confiáveis. Estes resultados confirmam aqueles obtidos por Martinez et al. (2000), que, ao compararem os VGs (PTAs) de touros estimados pelo desempenho de suas progênies puras e mestiças, em separado e conjuntamente, observaram que um índice combinando os VGs obtidos de análises separadas era o método mais indicado para a seleção de touros.

Uma alternativa para o ajuste da heterogeneidade de variância entre os grupos genéticos, caso a estimativa de correlação genética entre eles fosse próxima a unidade, seria um modelo heterogêneo (sem estrutura multicaráter) igual ao ajustado por Varona et al. (1997), no estudo de heterogeneidade de variância entre sexos com bovinos da raça Pirenaica, utilizada para a produção de carne na Espanha. Este modelo é considerado por Gianola (1986) como mais parcimonioso, quanto ao número de parâmetros a serem estimados e eventualmente mais adequado, se

Tabela 7 - Correlações de ordem entre os valores genéticos para a produção de leite preditos pelos modelos ajustados para touros e vacas da raça Gir, por nível de confiabilidade $\left(\mathbf{r}_{\mathrm{ti}}\right)$ obtido com o modelo bicarater

Table 7 - Rank correlation between milk yield breeding values for purebred Gir bulls and cows predicted by the two models of analysis classified by accuracy of the breeding values obtained from the bivariate model

\begin{tabular}{crrrr}
\hline $\begin{array}{c}\text { Nível de } \\
\text { confiabilidade } \\
\text { Accuracy }\end{array}$ & \multicolumn{2}{c}{$\begin{array}{c}\text { Touros } \\
\text { Bulls }\end{array}$} & \multicolumn{2}{c}{$\begin{array}{c}\text { Vacas } \\
\text { Cows }\end{array}$} \\
\hline $0,0<\mathrm{r}_{\mathrm{ti}}<0,5$ & $(175)$ & 0,992 & $(850)$ & 0,997 \\
$0,5-0,6$ & $(43)$ & 0,996 & $(2321)$ & 0,992 \\
$0,6-0,7$ & $(35)$ & 0,992 & $(644)$ & 0,994 \\
$>0,7$ & $(85)$ & 0,966 & $(53)$ & 0,994 \\
Geral & $(338)$ & 0,987 & $(3868)$ & 0,993 \\
Overall & & & & \\
\hline
\end{tabular}

R. Bras. Zootec., v.33, n.3, p.555-563, 2004 a disponibilidade de dados (número e estrutura) compromete a precisão das estimativas dos componentes de (co)variância.

O modelo heterogêneo (Varona et al., 1997) requer o pré-ajustamento dos registros de produção de cada grupo genético (por exemplo para a estimativa do desvio-padrão fenotípico). Há questionamentos quanto ao pré-ajustamento (Costa, 2001), que em geral é aplicado assumindo-se a herdabilidade constante ou homogênea e a correlação genética igual a 1.0 entre as subclasses da fonte causadora de heterogeneidade de variância. Em adição, o préajustamento consiste apenas em ajuste de escala dos fenótipos e pode não ser adequado, devido à possível presença de vários outros fatores associados a heterogeneidade de variância que poderiam ser, então, ignorados (Ibáñez et al., 1996).

O ajuste utilizado nos Estados Unidos (Wiggans \& VanRaden, 1991) resulta em aumento do desviopadrão fenotípico em rebanhos-ano de regiões de baixa variância e vice-versa. A implementação deste ajuste resultou no uso de maior estimativa herdabilidade para rebanhos de maior variância, e o efeito geral foi redução na percentagem de vacas elite identificadas em rebanhos de maior variância e aumento na concentração de vacas elite nos rebanhos de menor variância. Entretanto, Meuwissen et al. (1996) e Robert-Granié et al. (1999) consideram mais apropriado um modelo de ajuste no qual a estimativa simultânea dos parâmetros de localização e dispersão das classes associadas à heterogeneidade de variância sejam realizadas simultaneamente às avaliações genéticas. Van Vleck (1987) concluiu que, em situações de diferentes herdabilidades e correlação genética entre ambientes inferior à unidade, os procedimentos com modelos mistos multicaráter podem ser usados para se obterem as melhores avaliações e otimizar a seleção de animais.

A presença da heterogeneidade de variância entre os grupos genéticos indica a necessidade do ajuste para evitar erros na classificação de touros e vacas nas avaliações genéticas. Na medida em que se considera que a estrutura dos dados e as estimativas obtidas no presente estudo correspondem, com satisfatória precisão, ao observado na população de Gir e seus mestiços, o modelo multicaráter, com estrutura de covariância conhecida, mostra-se uma alternativa adequada para ajuste da heterogeneidade de variância entre grupos genéticos puros e mestiços da raça Gir. 


\section{Conclusões}

Os efeitos da heterogeneidade de variância para as produções de leite e de gordura devem ser considerados nas avaliações genéticas de touros Gir, quando os seus grupos de progênie tem animais de diferentes composições raciais.

A distribuição adequada (homogênea) da progênie entre grupos determinantes da heterogeneidade de variância é importante para a seleção precisa dos touros de maior mérito genético.

No caso da seleção de vacas, a implementação de um modelo multicaráter como procedimento para ajuste da heterogeneidade de variância pode tornar a identificação de vacas elite mais precisa e, portanto, significar maior potencial de progresso genético pela escolha correta de mães de touros entre as vacas de maior mérito genético.

\section{Agradecimento}

À Associação Brasileira de Criadores de Gir Leiteiro - ABCGIL e à Coordenação do Programa Nacional de Melhoramento do Gir Leiteiro, pela disponibilização dos dados. Aos revisores ad hoc, pelas sugestões apresentadas.

\section{Literatura Citada}

BOLDMAN, K.G.; FREEMAN, A.E. Adjustment for heterogeneity of variances by herd level in dairy cow and sire evaluation. Journal of Dairy Science, v.73, n.2, p.503-512, 1990.

BOLDMAN, K.G.; KRIESE, L.A.; Van VLECK, L.D. et al. A manual for the use of MTDFREML: a set of programs to obtain estimates of variance and covariances (Draft). Maryland: USDA/Agricultural Research Service, 1995. 110p.

COSTA, C.N. Interação genótipo ambiente. In: VALENTE, J.; DURÃES, M.C.; MARTINEZ, M.L. et al. (Eds.) Melhoramento genético de bovinos de leite. Juiz de Fora: Embrapa Gado de Leite, 2001. p.151-179.

COSTA, C.N. An investigation into heterogeneity of variance for milk and fat yields of Holstein cows in Brazilian herd environments. Genetics and Molecular Biology, v.22, n.3, p.375-381, 1999.

DONG, M.; MAO, I.L. Heterogeneity of (Co)variance and heritability in different levels of intraherd milk production variance and of herd average. Journal of Dairy Science, v.73, n.3, p.438-444, 1990.

GIANOLA, D. On selection criteria and estimation of parameters when the variance is heterogeneous. Theoretical and Applied Genetics, v.72, n.5, p.671-677, 1986.
GARRICK, D.J.; POLLAK, E.J.; QUAAS, R.L. et al. Variance heterogeneity in direct and maternal weight traits by sex and percent purebred for Simmental-sired calves. Journal of Animal Science, v.73, n.10, p.2515-2525, 1989.

IBÁÑEZ, M.A.; CARABAÑO, M.J.; FOULLEY, J.L. et al. Heterogeneity of herd-year phenotypic variances in the Spanish Holstein-Friaisn cattle: sources of heterogeneity and genetic evaluation. Livestock Production Science, v.45, n.2/3, p.137-147, 1996.

MACHADO, C.H.C.; COSTA, C.N. A contribuição da ABCZ para o melhoramento genético de zebuínos no Brasil. In: MINAS LEITE, 2., 2000, Juiz de Fora.Anais...Juiz de Fora: Embrapa Gado de Leite, 2000. p.41-45.

MARTINEZ, M.L.; VERNEQUE, R.S.; TEODORO, R.L. et al. Relações entre as capacidades preditas de transmissão de touros Gir e a produção de leite de suas filhas puras e mestiças. Revista Brasileira de Zootecnia, v.29, n.3, p.692-699, 2000

MEUWISSEN, T.H.E.; DE JONG, G.; ENGEL, B. Joint estimation of breeding values and heterogeneous variances of large data files. Journal of Dairy Science, v.79, n.2, p.310-316, 1996.

POWELL, R.L.; NORMAN, H.D.; WEILAND, B.T. Cow evaluation at different milk yield of herds. Journal of Dairy Science, v.66, n.1, p.148-154, 1983.

ROBERT-GRANIÉ, C.; BONAITI, B.; BOICHARD, D. et al. Accounting for variance heterogeneity in French dairy cattle genetic evoluation. Livestock Production Science, v.60, n.2/3, p.343-357, 1999.

RODRIGUEZ-ALMEIDA, F.; Van VLECK, L.D.; CUNDIFF, L.V. et al. Heterogeneity of variance by sire breed, Sex, and dam breed in 200- and 365-day weights of beef cattle from a top cross experiment. Journal of Animal Science, v.73, n.9, p.2579-2588, 1995.

Van VLECK, L.D. Selection when traits have different genetic and phenotypic variances in diffeent environments.Journal of Dairy Science, v.70, n.2, p.337-344, 1987.

VARONA, L.; MORENO, C.; GARCIA-CORTES, L.A. et al. Model determination in a case of heterogeneity of variance using sampling techniques. Journal of Animal Breeding and Genetics, v.114, n.1, p.1-12, 1997.

VERNEQUE, R.S.; LEDIC, I.L.; MARTINEZ, M.L. et al. Programa de melhoramento do Gir Leiteiro. In: SIMPÓSIO NACIONAL DA SOCIEDADE BRASILEIRA DE MELHORAMENTO ANIMAL, 3., 2000, Belo Horizonte. Anais... Belo Horizonte: Sociedade Brasileira de Melhoramento Animal, 2000. p.212-218.

VINSON, W.E. Potential biases in genetic evaluations from differences in variation within herds. Journal of Dairy Science, v.70, n.11, p.2450-2455, 1987.

WIGGANS, G.R.; VANRADEN, P.M. Method and effect of adjustment of heterogeneous variance. Journal of Dairy Science, v.74, n.12, p.4350-4357, 1991.

Recebido em: 12/09/02 Aceito em: 02/10/03 